\title{
Modified method for proximate analysis of biochars
}

\author{
Deborah Aller, Santanu Bakshi, David Laird* \\ Department of Agronomy, Iowa State University, Ames, IA 50011, USA
}

E-mail address: dmaller@iastate.edu (D. Aller)

E-mail address: santanubakshi@gmail.com (S. Bakshi)

E-mail address: dalaird@iastate.edu (D. Laird)

*Corresponding author at: Department of Agronomy, Iowa State University, Ames, IA 50011, USA. Tel.: +1-515-294-1581

E-mail address: dalaird@iastate.edu (D. Laird) 


\begin{abstract}
:
Proximate analysis is widely used to determine moisture, volatile matter (VM), fixed carbon (FC) and ash content of biochars. The original ASTM D1762-84 method was developed to assess quality of hardwood charcoal for use as fuel. We have developed a modified proximate analysis method to assess quality of diverse biochars for use as soil amendments. We determined that a $\mathrm{N}_{2}$ purge is necessary during both moisture and VM determination to avoid errors associated with sample oxidation. We assessed a range of boundary temperatures $\left(350-950^{\circ} \mathrm{C}\right)$ for separating $\mathrm{VM}$ and $\mathrm{FC}$, and determined that $800^{\circ} \mathrm{C}$ is the minimum temperature required to distinguish between VM and FC in biochars. Furthermore, correlation between $\mathrm{VM} / \mathrm{FC}$ and molar $\mathrm{H} / \mathrm{C}_{\text {org }}$ ratios suggests that $\mathrm{VM} / \mathrm{FC}$ ratios are a useful measure of biochar stability. Use of the proposed modified method is encouraged to reduce variance in analytical results among studies.
\end{abstract}

Keywords: proximate analysis; volatile matter; fixed carbon; $\mathrm{N}_{2}$ Purge; biochar stability; $\mathrm{VM} / \mathrm{FC}$ ratio 


\section{Introduction}

Proximate analysis, American Society for Testing and Materials (ASTM) method number D1762-84, was originally developed for analysis of wood charcoal [1]. More recently, however, this method has been utilized for assessing quality of biochar, the solid co-product of thermochemical conversion of biomass to bioenergy, which is applied to soils to enhance soil quality and sequester carbon. Proximate analysis provides basic characterization information about biochars including moisture, volatile matter (VM), fixed carbon (FC) and ash content. Ash content is related to liming value and inorganic element content of biochar [2], while VM and FC have been used to estimate the labile and recalcitrant biochar fractions, respectively [3].

Biochars have many unique properties relative to charcoal. Most charcoals are produced from hardwood feedstocks by slow pyrolysis and are marketed in either lump or briquette forms for use as a fuel. Biochars, on the other hand, are produced from both wood and herbaceous feedstocks such as, corn stover, rice husks, sugarcane bagasse, and switchgrass, using various thermochemical conversion technologies, including slow pyrolysis, fast pyrolysis and gasification. Biochars are intended for use as soil amendments and vary widely in particle size, chemical composition and porosity; hence their impacts on agro-ecosystems can be highly variable [4].

Several factors influence the physical and chemical properties of biochars. Brewer et al. [5] conducted an extensive biochar characterization study and reported that the chemical and physical properties of biochar differ depending on both production technique and biomass feedstock. Other studies indicate that biochar properties change over time 
(weathering or aging) in soil environments $[6,7]$ and thus both fresh and aged biochars must be studied $[8,9,10]$. Lastly, Bradbury and Shafidazeh [11] reported weight gain by biochar during moisture determination using standard oven drying techniques, implying $\mathrm{O}_{2}$ chemisorption on biochar surfaces. The diversity of biochar properties relative to charcoal and the evidence for chemisorption of $\mathrm{O}_{2}$ on biochar surfaces suggest a need to evaluate the reproducibility and/or utility of results obtained using the ASTM proximate analysis method for biochars.

The ASTM method stipulates that crucibles and lids be heated to $750^{\circ} \mathrm{C}$, cooled, and weighed prior to their use for proximate analysis. Samples are initially sieved to $850 \mu \mathrm{m}$, then moisture content is determined as the percent mass loss on heating air-dry samples to $105^{\circ} \mathrm{C}$ in an air atmosphere, $\mathrm{VM}$ as percent mass lost between $105^{\circ} \mathrm{C}$ and $950^{\circ} \mathrm{C}$, and percent ash as mass remaining after combustion at $750^{\circ} \mathrm{C}$ for 6 hours. During VM determination the ASTM method prescribes a process of preheating the furnace to $950^{\circ} \mathrm{C}$, preheating the samples in crucibles (lids-on) by placing them on the outer edge of the furnace with the door open for two minutes at $300^{\circ} \mathrm{C}$, then moving them a little farther into the furnace with the door open for three minutes at $500^{\circ} \mathrm{C}$, and finally placing the crucibles at the back of the furnace with the door closed for six minutes.

We consider the ASTM procedure to be problematic because the samples may be exposed to $\mathrm{O}_{2}$ during both the moisture and VM determination steps. Furthermore, the ASTM VM procedure is not well controlled, as heating is not necessarily the same in all furnaces when the door is open, and moving samples around in a hot furnace limits the number of samples that can be analyzed at the same time, and is potentially hazardous. 
Many biochar research groups have chosen to modify the ASTM proximate analysis method [5, 8, 12-18]. None of these researchers, however, have systematically investigated the effects of method modifications on the results. Following is a brief discussion of modified proximate analysis procedures used by Mitchell et al. [12] and Enders et al. [13], which were chosen to emphasize the variability of procedures used for proximate analysis found in the literature.

Mitchell et al. [12] utilized thermogravimetric analysis (TGA) for proximate analysis of biochars. Moisture content was estimated as percent of sample mass lost between room temperature and $100^{\circ} \mathrm{C}, \mathrm{VM}$ as percent mass lost between $150^{\circ} \mathrm{C}-450^{\circ} \mathrm{C}$, ash as percent mass remaining after the $750^{\circ} \mathrm{C}$ combustion, and $\% \mathrm{FC}$ as one hundred minus $\%$ moisture, \%ash, and \%VM. During the TGA procedure, samples were heated under a $\mathrm{N}_{2}$ atmosphere to $750^{\circ} \mathrm{C}$, then air was introduced into the system and maintained for four minutes to facilitate combustion. The use of a $\mathrm{N}_{2}$ purge for both moisture and VM determination should preclude weight gain due to chemisorption of $\mathrm{O}$, but the $450^{\circ} \mathrm{C}$ boundary temperature for VM determination has not been validated with diverse biochars. Other potential problems with the Mitchell et al. [12] method are a small samples size, which can decrease analytical precision, and the fact that many laboratories do not have access to a TGA instrument.

A detailed description of the modified procedure of Enders et al. [13] is provided in their supplementary material but in short, crucibles + covers were heated to $750^{\circ} \mathrm{C}$ and cooled to ensure no moisture was present prior to analysis. Moisture content was determined in an argon rich atmosphere as mass lost after 18 hours at $105^{\circ} \mathrm{C}$. Percent VM was determined without argon after heating the furnace to $950^{\circ} \mathrm{C}$, opening the door to put 
crucibles in the furnace, leaving samples for 10 minutes, and then removing and letting samples cool. Ash content was determined as mass remaining after 6 hours at $750^{\circ} \mathrm{C}$ in air. The authors highlighted that during VM determination the furnace cooled off when the door was opened and did not reach $950^{\circ} \mathrm{C}$ again for eight minutes. Although covers were on the crucibles during VM determination to reduce exposure to $\mathrm{O}_{2}$, opening the door introduced a large amount of oxygen into the furnace, increasing the potential for partial ashing of the samples.

The many variations of the proximate analysis method used by researchers is problematic for the biochar research community as the lack of an accepted standard protocol limits comparisons of results between studies. Proximate analysis is an important biochar characterization method because it is quick, easy, and relatively inexpensive and because it provides an estimation of the size of the ash, VM and FC labile and recalcitrant fractions in biochars. Assuming that VM and FC are indirect measures of the labile and recalcitrant biochar fractions, the results of proximate analysis are useful for predicting the impact of biochar amendments on soil properties and the long-term stability of biochar $\mathrm{C}$ in soil environments.

The goal of this study was to develop a modified proximate analysis procedure for biochars that is accurate, reproducible and simple. Specifically, we seek to develop a procedure that can be used with diverse biochar samples, reduces sample handling during the procedure (relative to the ASTM method), allows many samples to be analyzed at the same time and yields results which are relevant for assessing biochar $\mathrm{C}$ stability in soils.

\section{Materials and Methods:}


The source and abbreviations for the 22 biochars used in this study are listed in supporting materials (Table S1). Details of the production, collection, weathering and corresponding chemical and physical properties of the biochars are described elsewhere [7]. In brief, 6 un-weathered (Fresh-1), 6 laboratory-weathered (LW) ${ }^{1}, 5$ un-weathered but stored (Fresh-2), and 5 field-weathered (FW) ${ }^{1}$ biochars were investigated. These biochars were variously produced by fast pyrolysis, slow pyrolysis and gasification techniques from five different biomass feedstocks (corn stover, soybean residue, hardwood, switchgrass and macadamia nut shell). The controlled chemical aging procedure for the six LW biochars (particle size $<1 \mathrm{~mm}$ ) included a one month incubation at $40^{\circ} \mathrm{C}$ in a $1 \mathrm{M} \mathrm{HCl}$ solution with weekly additions of $30 \% \mathrm{H}_{2} \mathrm{O}_{2}$, followed by Ca-saturation, and then another one month incubation at $40^{\circ} \mathrm{C}$ in an aqueous solution containing dissolved organic carbon (compost tea). The five field weathered biochars were originally applied to experimental field plots in Minnesota and South Dakota in 2008 and 2011, respectively, and were collected and separated from soil in 2014. Initial bulk soil samples (0-20 cm depth) were air-dried and sieved $(44 \mathrm{~mm})$ to remove large biochar particles. Secondary sieving to separate smaller biochar particles occurred by wet sieving $(0.045 \mathrm{~mm})$ and hand picking. All field weathered biochar was subsequently ground using a mortar and pestle to a particle size of $<1 \mathrm{~mm}$ and stored in sealed containers [7]. $\mathrm{H}$ and $\mathrm{C}_{\text {org }}$ content of the various biochars was determined using a combustion analyzer (Vario Microcube, Elementar Analysensysteme GmbH) after the samples had been ball milled to reduce particle size and treated for $24 \mathrm{~h}$ with $0.05 \mathrm{~mol}$ $\mathrm{L}^{-1} \mathrm{HCl}$ to remove carbonates [7].

\footnotetext{
${ }^{1}$ Laboratory-weathered (LW) and field-weathered (FW) are equivalent to Laboratory-aged (LA) and Fieldaged (FA), respectively as found in Bakshi et al. [7].
} 


\subsection{Modified Proximate Analysis Method}

Moisture content of samples was determined based on mass loss after two hours at $105^{\circ} \mathrm{C}$ under $\mathrm{N}_{2}$ purge. Approximately $0.5 \mathrm{~g}$ of air-dried biochar was weighed into a ceramic crucible ( $\left.\mathrm{M}_{\text {initial-BC }}\right)$. The samples were placed inside of a Lindberg muffle furnace (model 51442), which was initially purged with $\mathrm{N}_{2}$ gas for $\geq 25$ minutes at a flow rate of $6 \mathrm{~L}$ $\min ^{-1}$, equivalent to roughly 10 furnace volumes, to ensure removal of all oxygen. During the subsequent heating phase the furnace was purged with $\mathrm{N}_{2}$ at a flow rate of $3 \mathrm{~L} \mathrm{~min}^{-1}$. After the 2 hours heating, the furnace was turned off and samples were transferred immediately to a desiccator, left to cool for one hour, and then weighed.

Volatile matter was determined by heating the oven dry samples under $\mathrm{N}_{2}$ purge to various potential $\mathrm{VM} / \mathrm{FC}$ separation temperatures. To determine the appropriate $\mathrm{VM} / \mathrm{FC}$ separation temperature, samples were heated at $2{ }^{\circ} \mathrm{C} \mathrm{min}^{-1}$ to each of nine different temperatures: $350^{\circ} \mathrm{C}, 550^{\circ} \mathrm{C}, 650^{\circ} \mathrm{C}, 700^{\circ} \mathrm{C}, 750^{\circ} \mathrm{C}, 800^{\circ} \mathrm{C}, 850^{\circ} \mathrm{C}, 900^{\circ} \mathrm{C}$, and $950^{\circ} \mathrm{C}$. During heating, the crucibles containing the biochar were covered with ceramic lids, placed in a stainless steel box inside of a muffle furnace (Thermo Scientific Lindberg/Blue M Box Furnace BF51894C-1). A N $\mathrm{N}_{2}$ purge line and thermocouple were inserted through the top of the furnace and down into the stainless steel box through a small hole in the box cover. The box was purged with $\mathrm{N}_{2}$ gas for $\geq 15$ minutes at a flow rate of $6 \mathrm{~L} \min ^{-1}$ (approximately 10 box volumes). After the initial purge, the $\mathrm{N}_{2}$ flow rate was decreased to $3 \mathrm{~L} \mathrm{~min}^{-1}$, the furnace was set to the desired peak separation temperature, and turned on. The temperature inside of the stainless steel box was measured every 60 seconds during the heating treatments. Once the temperature inside of the stainless steel box reached the desired $\mathrm{VM} / \mathrm{FC}$ separation temperature, the furnace was switched off and furnace door opened. The 
$\mathrm{N}_{2}$ purge inside the stainless steel box was maintained $\left(3 \mathrm{~L} \mathrm{~min}^{-1}\right)$ during cool down (24hrs), after which the crucibles were weighed to determine the mass of $\mathrm{FC}+\mathrm{Ash}\left(\mathrm{M}_{\mathrm{FC}+\mathrm{Ash}}\right)$ after subtracting the empty crucible weight.

Ash content of biochars was determined by heating the same samples to $730^{\circ} \mathrm{C}$ in an air atmosphere using the same muffle furnace. To ensure complete combustion, crucible lids were removed and a low flow of house air $\left(1.5 \mathrm{~L} \mathrm{~min}^{-1}\right)$ was constantly flushed through the furnace. The furnace was heated to $730^{\circ} \mathrm{C}$ and held at that temperature overnight $(8-10$ hours). After ashing, the furnace was switched off and allowed to cool for one hour before the samples were transferred to a desiccator to cool. The crucibles were weighed and ash mass $\left(\mathrm{M}_{\text {ash }}\right)$ was determined by subtracting the empty crucible weight.

All reported proximate analysis data in this manuscript are the arithmetic mean of triplicate measurements.

\subsection{Proximate Analysis Calculations}

The percent moisture (\% Moisture) and percent ash (\% Ash) were determined using equations 1 and 2, respectively.

$$
\begin{aligned}
& \% \text { Moisture }=\left(\frac{\left(\mathrm{M}_{\text {initial }}-M_{O D-B C}\right)}{M_{O D-B C}}\right) * 100 \\
& \% \text { Ash }=\left(\frac{M_{a s h}}{M_{O D-B C}}\right) * 100
\end{aligned}
$$

where $\mathrm{M}_{\text {initial }}$ is the initial sample mass in grams, $\mathrm{M}_{\mathrm{OD}-\mathrm{BC}}$ is the mass in grams of the sample after oven drying at $105^{\circ} \mathrm{C}$ under an $\mathrm{N}_{2}$ purge, and $\mathrm{M}_{\text {ash }}$ is the sample mass in grams after combustion at $730{ }^{\circ} \mathrm{C}$ overnight. 
The percent volatile matter $(\% \mathrm{VM})$ and percent ash free volatile matter (\% $\mathrm{VM}_{\text {ash- }}$ free) were determined using equations 3 and 4, respectively.

$\% V M=\left(\frac{M_{O D-B C}-M_{F C+A s h}}{M_{O D-B C}}\right) * 100$

$\% V M_{a s h-f r e e}=\left(\frac{M_{O D-B C}-M_{F C+A s h}}{M_{O D-B C}-M_{a s h}}\right) * 100$

where $\mathrm{M}_{\mathrm{FC}+\mathrm{Ash}}$ is the mass in grams of fixed carbon and ash in the sample.

The percent fixed carbon $(\% \mathrm{FC})$ and percent ash free fixed carbon $\left(\% \mathrm{FC}_{\text {ash-free }}\right)$ were determined using equations 5 and 6, respectively, as previously described by Ronsse et al. [19].

$\% F C=\left(\frac{\left(M_{F C+a s h}-M_{a s h}\right)}{\left(M_{O D-B C}\right)}\right) * 100$
$\% F C_{a s h-f r e e}=\left(\frac{\left(M_{F C+a s h}-M_{a s h}\right)}{\left(M_{O D-B C}-M_{a s h}\right)}\right) * 100$

\subsection{Statistical Analysis}

All analyses were carried out in SAS 9.4 (SAS Institute Inc., 2013), with statistical significance accepted at the 5\% significance level. An initial t-test was conducted to determine whether results differed between the ASTM method and the Modified method. A significant method by biochar type interaction was found $(P<0.05)$. Analysis of variance (ANOVA) was subsequently conducted to assess differences between methods on each individual biochar type, with biochar type referring to the production technique, feedstock, and weathering treatments (Fresh-1, LW, Fresh-2, FW) of the 22 different biochars used in this study. 


\section{Results and discussion}

\subsection{Assessment of moisture and ash contents}

The moisture content of the 22 diverse biochars varied substantially and was influenced by the degree of weathering (Fig. 1). Overall the LW biochars had significantly higher moisture content than Fresh-1, Fresh-2, and FW biochars with the exception of FW HS2 and both Fresh-2 and FW MNS $(P<0.05)$. This may have resulted from the intensive oxidation and acidification treatments, which transformed the surfaces of the LW biochars from being hydrophobic to hydrophilic, by the incomplete drying of the LW biochars prior to storage, and/or the absorption of moisture during storage. Furthermore, there was generally more variability in moisture content of the LW biochars than the Fresh-1, Fresh2, and FW biochars. This suggests non-homogeneity in moisture content among biochar subsamples taken from the storage containers.

Statistical analysis of the moisture content data indicated a significant biochar type by method interaction $(P<0.05)$. The results indicate that the ASTM and Modified methods gave different results for moisture content in 9 of the 22 biochars, and that differences in moisture content values determined by the two methods are not consistent but rather influenced by biochar feedstock, weathering, and production technique (Fig. 1).

The Modified method indicated higher moisture levels than the ASTM method for all six of the Fresh-1 biochars, with these values significantly higher in five of the six biochars $(P<0.05)$. Fresh-1 CF was the only biochar found to be not significantly different for moisture content between the two methods $(P=0.064)$. For both methods moisture content is determined by heating the samples to $105^{\circ} \mathrm{C}$ for 2 hours, with the key difference 
between the methods being use of an $\mathrm{N}_{2}$ purged atmosphere in the Modified method and an air atmosphere in the ASTM method. Exposure to $\mathrm{O}_{2}$ during heating to $105^{\circ} \mathrm{C}$ could cause weight loss if thermally labile organic compounds are fully oxidized to $\mathrm{CO}_{2}$ and/or $\mathrm{H}_{2} \mathrm{O}$. Alternatively, exposure to $\mathrm{O}_{2}$ during heating to $105^{\circ} \mathrm{C}$ could cause weight gain if $\mathrm{O}_{2}$ is chemisorbed on biochar surfaces, due to partial rather than complete oxidation of biochar C. The Fresh-1 biochars were produced a few weeks prior to analysis and stored in sealed containers, thus they had minimal exposure to atmospheric $\mathrm{O}_{2}$, moisture, and other environmental conditions between production and analysis. Our results (Fig. 1) suggest that chemisorption of $\mathrm{O}_{2}$ significantly reduced the weight loss (apparent moisture content as determined by the ASTM method relative to the Modified method) for five of the six Fresh1 biochars. Indeed, two of the Fresh-1 biochars had significant "negative" moisture content (i.e., weight gain during heating to $105^{\circ} \mathrm{C}$ in air) as determined by the ASTM method (Table $\mathrm{S} 2$ ), which is strong evidence of $\mathrm{O}_{2}$ chemisorption.

In contrast with the results for the Fresh-1 biochars, there were no significant differences in measured moisture content between the two methods for the 5 Fresh-2 biochars, with the exception of Fresh-2 SG $(P=0.0008)$. We do not know details of storage conditions for the Fresh-2 biochars, but we do know that they were stored for $>3$ years, and based on the results (Fig. 1) it appears that these samples were variously exposed to $\mathrm{O}_{2}$ and moisture during storage, which may have limited the potential for chemisorption of $\mathrm{O}_{2}$ during heating.

The results are variable among the LW and FW biochars; 2 samples showed higher moisture content by the ASTM method, 1 showed higher moisture content by the Modified method, and 8 showed no significant difference in moisture content between the methods 
(Fig. 1). Again we attribute these differences to the exposure to $\mathrm{O}_{2}$ that occurs during drying in the ASTM method but not the Modified method. Lower apparent moisture levels by the ASTM method than the Modified method again implies chemisorption of $\mathrm{O}_{2}$. Higher apparent moisture levels by the ASTM method than the Modified method indicates mass loss as either $\mathrm{CO}_{2}$ or other volatile compounds. Both the $\mathrm{LW}$ and $\mathrm{FW}$ biochars had an opportunity to adsorb biogenic dissolved organic compounds (DOC) during aging [7], hence it is possible that differences in apparent moisture content determined by the ASTM and Modified methods are related to the presence of thermally labile biogenic organic compounds adsorbed on the surfaces of the LW and FW biochars.

The hydrophobicity of biochar surfaces and the degree of biochar weathering influence the ability of biochars to absorb water from an atmosphere or solution and the amount of water held by biochars when they are air dried. The lack of uniformity across biochars in retaining water has the potential to impact proximate analysis results. This highlights the need to standardize the reporting of proximate analysis moisture, VM, FC, and ash content data based on the oven dry weight of biochars. Additionally, any inaccuracy in determining the oven dry weight will impact the accuracy of subsequent determinations of VM, FC, and ash content of biochars. Our results for moisture determination (Fig. 1) emphasize the importance of determining moisture content of biochars in an atmosphere absent of $\mathrm{O}_{2}$. The use of a $\mathrm{N}_{2}$ purge in the Modified procedure prevented both complete oxidation of organic compounds to $\mathrm{CO}_{2}$ and the chemisorption of $\mathrm{O}_{2}$ onto biochar surfaces.

Both methods indicated similar estimates of ash content for 16 of the 22 biochars. Measured ash content values differed between methods for the Fresh-1 HF, Fresh-1 SF, 
Fresh-1 SS, LW HF, LW SF, and FW CG biochars (Fig. 2). There are no obvious connections between these six biochars that explain the divergent results. Moreover, two of these biochars were found to have higher ash content and four to have lower ash content as determined by the ASTM method relative to the Modified method. The protocols for determining ash content are similar for both methods, although in the Modified method a small amount of house air is constantly introduced into the furnace during ashing to ensure an adequate supply of $\mathrm{O}_{2}$ for complete combustion. With the ASTM method we observed that some biochar particles were resistant to oxidation, especially when encrusted in ash. Hence, incomplete combustion could explain the higher ash content measured by the ASTM method for two of the samples but not the lower ash content measured by the ASTM method for the four other samples relative to ash content measured by the Modified method. Differences in the extent of thermal degradation of mineral phase [15, 20], is another possible explanation for the observed differences in biochar ash content determined by the two methods. It is tempting to attribute these differences to sampling error and/or lack of homogeneity in the stored biochar samples, however, the ash analysis was determined in triplicate and standard errors are small relative to the difference between the methods for these six samples (Fig. 2). Overall, to ensure that all carbon is oxidized we recommend ashing samples at $730^{\circ} \mathrm{C}$ for an $8-10$ hour period with a constant low flow of air, to supply $\mathrm{O}_{2}$.

\subsection{Evaluation of $V M, F C$, and the $V M / F C$ separation temperature boundary}

Standardizing the method for determining VM and FC was a key objective of this study. To do so, we evaluated nine different VM/FC separation temperatures all under $\mathrm{N}_{2}$ 
purge to preclude any mass loss or gain due to oxidation. Our results indicate that mass loss on heating increased rapidly for all biochars between $350^{\circ} \mathrm{C}-650^{\circ} \mathrm{C}$, slowly between $650^{\circ} \mathrm{C}-800^{\circ} \mathrm{C}$, and then stabilized at and above $800^{\circ} \mathrm{C}$ (Fig. S1). Furthermore, the lack of change in apparent $\mathrm{VM} / \mathrm{FC}$ ratios relative to $\mathrm{VM} / \mathrm{FC}$ ratios determined for a $950^{\circ} \mathrm{C}$ separation temperature supports $800^{\circ} \mathrm{C}$ as the minimum temperature that should be used to determine VM content of biochars, although this does not preclude the use of higher temperatures (Fig. S2).

The importance of using an inert gas purge during VM determination is evident by comparing the $\% \mathrm{VM}$ results determined by the ASTM protocol and the Modified method using the $800^{\circ} \mathrm{C} \mathrm{VM/FC} \mathrm{separation} \mathrm{temperature} \mathrm{(Fig.} \mathrm{3).} \mathrm{Overall,} \mathrm{\% VM} \mathrm{differed} \mathrm{for} 16$ of the 22 biochars between the two methods $(P<0.05)$. Estimates of $\% \mathrm{VM}$ obtained with the ASTM method were significantly higher for all of the Fresh-1 and LW biochars except for LW HS1 and LW SS relative to the Modified method. By contrast, variable results were obtained for the Fresh-2 and FW biochars, with \%VM being higher when determined by the ASTM method for biochars produced by slow pyrolysis and lower for biochars produced by gasification relative to VM determined by the Modified method. These differences are influenced to some extend by the inaccurate estimates of oven dry weights and moisture content obtained by the ASTM method, however, we primarily attribute the opposing trends to exposure to $\mathrm{O}_{2}$ during VM determination by the ASTM method. The ASTM method relies on loose fitting lids on the crucibles and the release of volatile compounds from the sample to keep $\mathrm{O}_{2}$ away from the sample during heating for $\mathrm{VM}$ determination. With the ASTM protocol some sample exposure to $\mathrm{O}_{2}$ is inevitable. By contrast the Modified method uses a thorough $\mathrm{N}_{2}$ purge to preclude exposure of the samples 
to $\mathrm{O}_{2}$ during heating and cooling of samples. Our data suggest that a substantial amount of mass was lost (presumably as $\mathrm{CO}_{2}$ and $\mathrm{H}_{2} \mathrm{O}$ ) from the fast and slow pyrolysis biochars due to oxidation by $\mathrm{O}_{2}$ that leaked into the crucibles under the ASTM protocol. The greater aromaticity of gasification biochars likely resulted in only partial oxidation rather than complete oxidation and hence chemisorption of $\mathrm{O}_{2}$. This suggests that among the gasification biochars competing reactions which have a counter balancing effect are simultaneously taking place. The substantial differences between \%VM determined by the ASTM and Modified methods (Fig. 3) highlight the importance of using an inert gas purge when determining VM of biochars.

Percent FC values determined by the ASTM method differed $(P<0.05)$ from $\%$ FC values determined by the Modified method in 16 of 22 biochars (Fig. 4); revealing the exact opposite pattern as seen in \%VM (Fig. 3). Specifically, \%FC was lower when determined by the ASTM method for all fast and slow pyrolysis biochars with the exception of LW HS1, which was not significantly different. In contrast, the ASTM method indicated higher $\%$ FC values for the gasification biochars than the Modified method (Fig. 4). We again attribute these large differences in $\% \mathrm{FC}$ between the two methods to exposure to $\mathrm{O}_{2}$ during VM/FC determination following the ASTM protocol and differing levels of oxidation of biochar C.

\subsection{Assessment of proximate analysis as a method to measure biochar $C$ stability}

The ability to accurately estimate biochar $\mathrm{C}$ stability is critical to advance biochar utilization. Currently, the International Biochar Initiative (IBI) recommends the use of the molar $\mathrm{H} / \mathrm{C}_{\text {org }}$ ratio as an index of biochar $\mathrm{C}$ stability $[13,21]$. The $\mathrm{H} / \mathrm{C}_{\text {org }}$ ratio is also an 
index of biochar aromaticity as $\mathrm{H}$ is only bonded with $\mathrm{C}$ atoms on the edges of condensed aromatic $\mathrm{C}$ (graphitic) sheets. Thus the more disordered the biochar $\mathrm{C}$ structure, the more $\mathrm{H}$ it will contain and in theory the greater the rate of biochar degradation in soil environments. Elemental analysis of $\mathrm{H}$ and $\mathrm{C}$ content of biochars requires access to a thermal combustion instrument, which many laboratories do not have. Proximate analysis has the potential to be a less expensive and more widely available method for assessing biochar $\mathrm{C}$ stability and overall biochar quality, and hence provide useful input parameters into cropping systems models.

Previous work has reported a relatively strong correlation between \%VM and O:C elemental ratios of biochar but no evidence of a correlation between \% VM and estimated half-life of biochar $\mathrm{C}[22]$. For this reason, the IBI did not recommend the use of \%VM as an index of biochar C stability [21]. Percent VM, however, is strongly influenced by ash content $[\% \mathrm{VM}=\mathrm{VM} \times 100 /(\mathrm{VM}+\mathrm{FC}+\mathrm{Ash})]$, which is more a function of feedstock quality than pyrolysis technology and peak pyrolysis temperature. Furthermore, our results (Fig. 3) demonstrate that \%VM is highly dependent on proximate analysis methodology and that the ASTM method can both over and under estimate \%VM. However, previous work did not consider the importance of methodology used in evaluating proximate analysis data for assessing biochar C stability. Here we compared VM/FC ratios determined by the ASTM and Modified methods with $\mathrm{H} / \mathrm{C}_{\text {org }}$ ratios determined by thermal combustion analysis. VM/FC ratios are independent of ash content and hence a more robust index of biochar C stability than \%VM. 
The VM/FC ratios determined by both the ASTM and Modified methods are correlated $\left(\mathrm{R}^{2}=0.42\right.$ and 0.62 , respectively) with the $\mathrm{H} / \mathrm{C}_{\text {org }}$ ratios (Figs. 5a and $\left.5 \mathrm{~b}\right)$. The VM/FC ratios determined by the ASTM method are an order of magnitude higher $(P<$ 0.05) for all fast and slow pyrolysis biochars compared to $\mathrm{VM} / \mathrm{FC}$ ratios determined by the Modified method. By contrast, the VM/FC ratios for all the gasification biochars were lower when determined by the ASTM method than the Modified method. This is attributed to the opposing responses of the pyrolysis and gasification biochars to the exposure to limited $\mathrm{O}_{2}$ during VM determination in the ASTM method. As discussed previously, our data suggest that during the ASTM procedure the fast and slow pyrolysis biochars had greater mass lost as volatiles compared to the Modified method due to complete oxidation (presumably as $\mathrm{CO}_{2}$ and $\mathrm{H}_{2} \mathrm{O}$ ) whereas the gasification biochars had lower mass lost as volatiles under the ASTM method than the Modified method as a result of partial oxidation (chemisorption of $\mathrm{O}_{2}$ ).

In developing the Modified method, we considered the relationship between $\mathrm{VM} / \mathrm{FC}$ and $\mathrm{H} / \mathrm{C}_{\text {org }}$ ratios for potential $\mathrm{VM} / \mathrm{FC}$ separation temperatures ranging from 350 to $950^{\circ} \mathrm{C}$ (Fig. S3). For VM/FC separation temperatures greater than $650^{\circ} \mathrm{C}$, the $\mathrm{VM} / \mathrm{FC}$ and $\mathrm{H} / \mathrm{C}_{\text {org }}$ ratios of the studied biochars are correlated and show similar distributions for the fast pyrolysis, slow pyrolysis and gasification biochars. The gasification biochars have relatively low $\mathrm{H} / \mathrm{C}_{\text {org }}$ and $\mathrm{VM} / \mathrm{FC}$ ratios, which is consistent with greater condensation into polyaromatic $\mathrm{C}$ structures. The fast pyrolysis biochars have relatively high $\mathrm{H} / \mathrm{C}_{\text {org }}$ and $\mathrm{VM} / \mathrm{FC}$ ratios, which is consistent with more single ring aromatic structures and/or the condensation of volatile aliphatic compounds into the biochars during fast pyrolysis. The slow pyrolysis biochars are clustered in the middle between the fast pyrolysis and 
gasification biochars on the $\mathrm{H} / \mathrm{C}_{\text {org }}$ and $\mathrm{VM} / \mathrm{FC}$ plots. These data suggest that the $\mathrm{H} / \mathrm{C}_{\text {org }}$ and $\mathrm{VM} / \mathrm{FC}$ ratios are related measures of stability but are not identical. Differences between the two indices of biochar stability are attributed to the inherent differences among biochars which result from the use of various feedstocks and biochar production techniques. These findings suggest that $\mathrm{VM} / \mathrm{FC}$ ratios as determined by the Modified proximate analysis method have relevance for assessing the long-term stability of biochar $\mathrm{C}$ in soils. Specifically, \%VM when measured using an inert gas purge, which avoids problems with partial or complete oxidation, is by definition a measure of the mass of the thermally labile biochar fraction. Further research is needed to determine whether or not the thermally labile fraction is comparable to the biologically labile biochar fraction.

\section{Conclusions}

Significant differences were found between the ASTM method and Modified method. Results from our Modified method support its appropriateness for use in the proximate analysis of biochars and for $\mathrm{VM} / \mathrm{FC}$ ratios to assess biochar $\mathrm{C}$ stability and quality. The Modified method accommodates a large sample size, reduces sample handling and potential hazards, is applicable for diverse biochars, and avoids errors caused by oxidation, which are inherent to the ASTM method. A standard proximate analysis protocol designed specifically with biochar diversity in mind will minimize differences in results among studies and facilitate the greater comparison of biochar properties between researchers. 


\section{Acknowledgements}

This work was made possible by the Agriculture and Food Research Initiative Competitive Grant 2011-68005-30411 from the USDA National Institute of Food and Agriculture and in part by the National Science Foundation under Grant Number EPS1101284. The authors would like to thank Dr. Kurt Spokas and Dr. Rajesh Chintala for providing the field weathered biochars and Samuel Rathke for assistance in the laboratory. The authors are also grateful for reviewer comments which improved the quality of this manuscript. 


\section{References}

[1] ASTM Standard D1762-84, Chemical Analysis of Wood Charcoal, ASTM International, West Conshohocken, PA, USA, 2007, http://dx.doi.org/10.1520/D1762-84R07

[2] R.B. Fidel, Biochar properties and impact on soil $\mathrm{CO}_{2}$ and $\mathrm{N}_{2} \mathrm{O}$ emissions, Dissertation. Iowa State University, 2015, http://lib.dr.iastate.edu/etd/14812/

[3] S. Archontoulis, I. Huber, F. Miguez, P. Thorburn, N. Rogovska, D. Laird, A model for mechanistic and system assessments of biochar effects on soils and crops and tradeoffs, GCB Bioenergy (2016) doi:10.1111/gcbb.12314

[4] P. Blackwell, G. Riethmuller, M. Collins, Biochar application to soil, in: J. Lehmann, S. Joseph (Eds.), Biochar for Environmental Management: Science and Technology, Earthscans, London, 2009, pp. 207-226.

[5] C. Brewer, K. Schmidt-Rohr, J. Satrio, R. Brown, Characterization of biochar from fast pyrolysis and gasification systems. Environ. Prog. Sustain. Energy 28 (2009) 386396. doi:10.1002/ep.10378

[6] A. Downie, A. Crosky, P. Munroe, Physical properties of biochar, in: J. Lehmann, S. Joseph (Eds.), Biochar for Environmental Management: Science and Technology, Earthscans, London, 2009, pp. 13-32.

[7] S. Bakshi, D. Aller, D. Laird, R. Chintala, Comparison of the physical and chemical properties of laboratory- and field-aged biochars, J. Environ. Qual. (2016) doi:10.2134/jeq2016.02.0062

[8] A. Mukherjee, A.R. Zimmerman, W.G. Harris, Surface chemistry variations among a series of laboratory-produced biochars, Geoderma 163 (2011) 247-255. doi:10.1016/j.geoderma.2011.04.021

[9] S. Hale. K. Hanley, J. Lehmann, A. Zimmerman, G. Cornelissen, Effects of chemical, biological, and physical aging as well as soil addition on the sorption of pyrene to activated carbon and biochar, Environ. Sci. Technol. 45 (2011) 10445-10453. doi: 10.1021/es202970x

[10] A. Cross, S. Sohi, A method for screening the relative long-term stability of biochar, GCB Bioenergy 5 (2013) 215-220. doi:10.1111/gcbb.12035

[11] A. Bradbury, F. Shafizadeh, Chemisorption of oxygen on cellulose char, Carbon 8 (1980) 109-116. doi:10.1016/0008-6223(80)90018-4 
[12] P. Mitchell, T. Dalley, R. Helleur, Preliminary laboratory production and characterization of biochars from lignocellulosic municipal waste, J. Anal. Appl. Pyrol. 99 (2013) 71-78. doi:10.1016/j.jaap.2012.10.025

[13] A. Enders, K. Hanley, T. Whitman, S. Joseph, J. Lehmann, Characterization of biochars to evaluate recalcitrance and agronomic performance, Bioresour. Technol. 114 (2012) 644-653. doi:10.1016/j.biortech.2012.03.022

[14] X. Peng, L. Ye, C. Wang, H. Zhou, B. Suna, Temperature- and duration-dependent rice straw-derived biochar: Characteristics and its effects on soil properties of an Ultisol in southern China, Soil Till. Res. 112 (2011) 159-166.

doi:10.1016/j.still.2011.01.002

[15] H.J. Bachmann, T.D. Bucheli, A. Dieguez-Alonso, D. Fabbri, H. Knicker, H.-P. Schmidt, A. Ulbricht, R. Becker, A. Buscaroli, D. Buerge, A. Cross, D. Dickinson, A. Enders, V.I. Esteves, M.W.H. Evangelou, G. Fellet, K. Friedrich, G. Gasco Guerrero, B. Glaser, U.M. Hanke, K. Hanley, I. Hilber, D. Kalderis, J. Leifeld, O. Masek, J. Mumme, M.P. Carmona, R. Calvelo Pereira, F. Rees, A.G. Rombolà, J.M. de la Rosa, R. Sakrabani, S. Sohi, G. Soja, M. Valagussa, F. Verheijen, F. Zehetner, Toward the standardization of biochar analysis: The COST action TD1107 interlaboratory comparison. J. Agric. Food Chem. 64 (2016) 513-527. doi:10.1021/acs.jafc.5b05055

[16] R. Calvelo Pereira, J. Kaal, M. Camps Arbestain, R. Pardo Lorenzo, W. Aitkenhead, M. Hedley, F. Macías, J. Hindmarsh, J.A. Maciá-Agulló, Contribution to characterization of biochar to estimate the labile fraction of carbon, Org. Geochem. 42 (2011) 1331-1342. doi.org/10.1016/j.orggeochem.2011.09.002

[17] O.R. Harvey, L.-J. Kuo, A.R. Zimmerman, P. Louchouarn, J.E. Amonette, B.E. Herbert, An index-based approach to assessing recalcitrance and soil carbon sequestration potential of engineered black carbons (biochars), Environ. Sci. Technol. 46 (2012) 1415-1421. doi:10.1021/es2040398

[18] T. Wang, M. Camps-Arbestain, M. Hedley, Predicting C aromaticity of biochars based on their elemental composition. Org. Geochem. 62 (2013) 1-6. doi.org/10.1016/j.orggeochem.2013.06.012

[19] F. Ronsse, S. Van Hecke, D. Dickinson, W. Prins, Production and characterization of slow pyrolysis biochar: influence of feedstock type and pyrolysis conditions, GCB Bioenergy, 5 (2013) 104-115. doi:10.1111/gcbb.12018 
[20] T. Wang, M. Camps-Arbestain, M. Hedley, B.P. Singh, R. Calvelo-Pereira, C. Wang, Determination of carbonate-C in biochars, Soil Research 52 (2014) 495-504. doi.org/10.1071/SR13177

[21] A. Budai, A. Zimmerman, A. Cowie, J. Webber, B. Singh, B. Glaser, C. Masiello, D. Andersson, F. Shields, J. Lehmann, M. Camps-Arbestain, M. Williams, S. Sohi, S. Joseph, Biochar carbon stability test method: an assessment of methods to determine biochar carbon stability. International Biochar Initiative. (2013) http://www.biocharinternational.org/sites/default/files/IBI_Report_Biochar_Stabilit y_Test_Method_Final.pdf

[22] K. Spokas, Review of the stability of biochar in soils: predictability of O:C molar ratios, Carbon Manag. 1 (2010) 289-303. doi:10.4155/cmt.10.32 


\section{Figure Captions}

Fig. 1 Percent moisture loss determined after two hours at $105^{\circ} \mathrm{C}$ for all biochars. An $\mathrm{N}_{2}$ purge was used with the Modified method but not with the ASTM method. Error bars represent one standard error of the mean.

Fig. 2 Percent ash content of all biochars determined by the ASTM and modified methods. Error bars represent one standard error of the mean.

Fig. 3 Percent volatile matter of all biochars determined by the ASTM and modified methods. Error bars represent one standard error of the mean.

Fig. 4 Percent fixed carbon (dry ash free) of all biochars for the ASTM and modified methods. Error bars represent one standard error of the mean.

Fig. 5 Volatile matter/fixed carbon $(\mathrm{VM} / \mathrm{FC})$ vs. $\mathrm{H} / \mathrm{C}_{\text {org }}(\mathrm{mol}: \mathrm{mol})$ ratios for all biochars grouped by production technique; a) VM and FC were determined using the ASTM method; b) VM and FC determined by our modified method using $800{ }^{\circ} \mathrm{C}$. 
Fig. 1

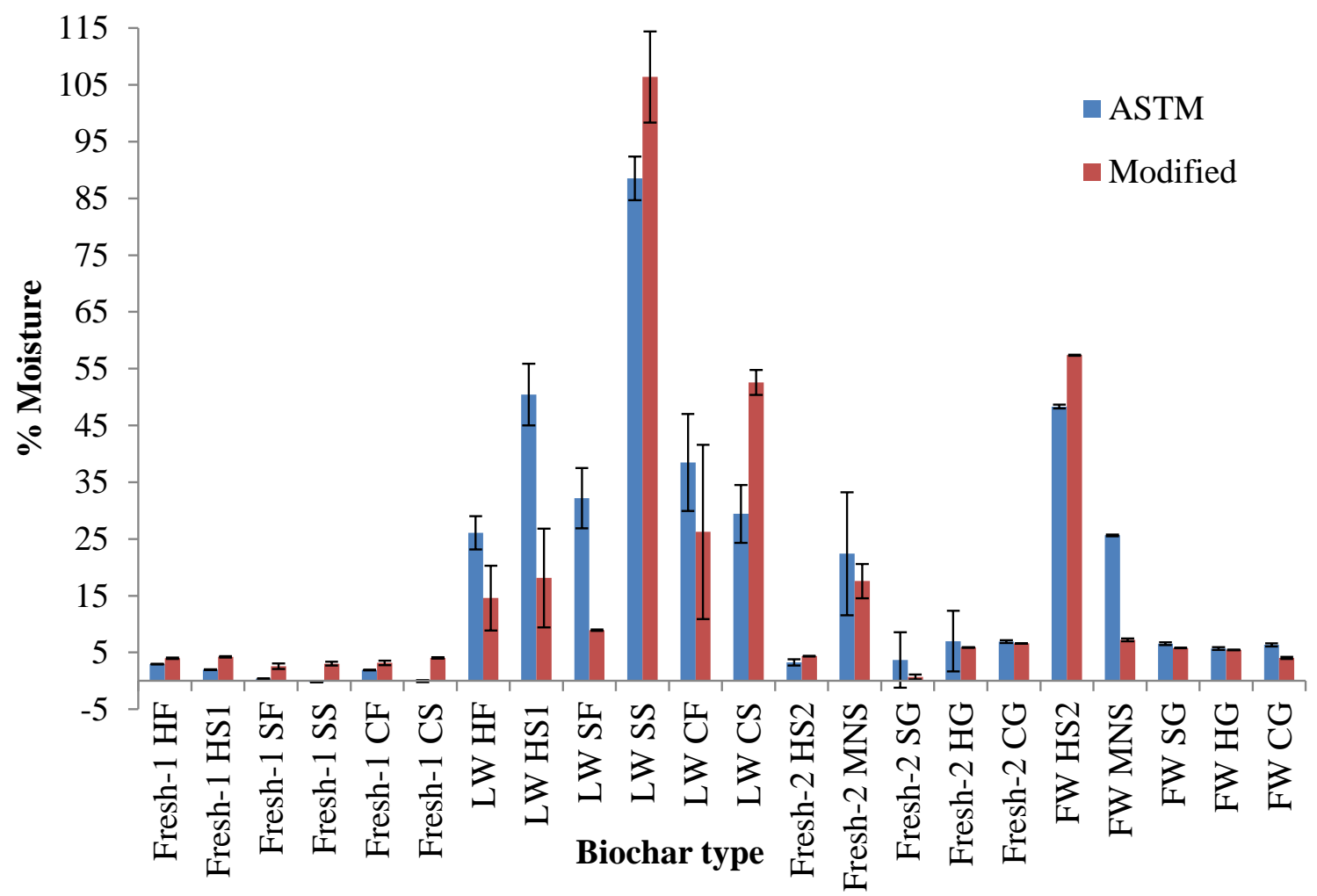


Fig. 2

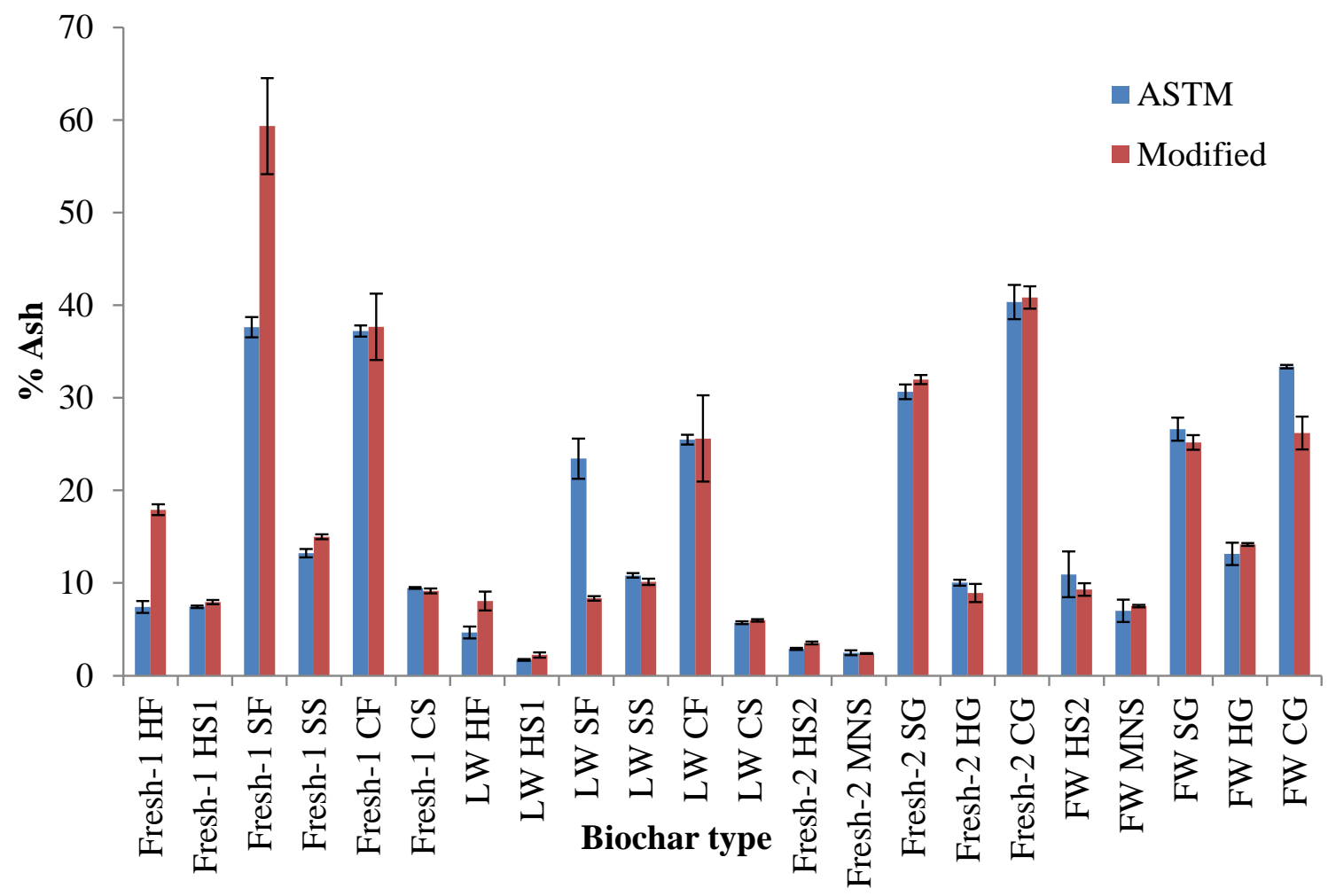


Fig. 3

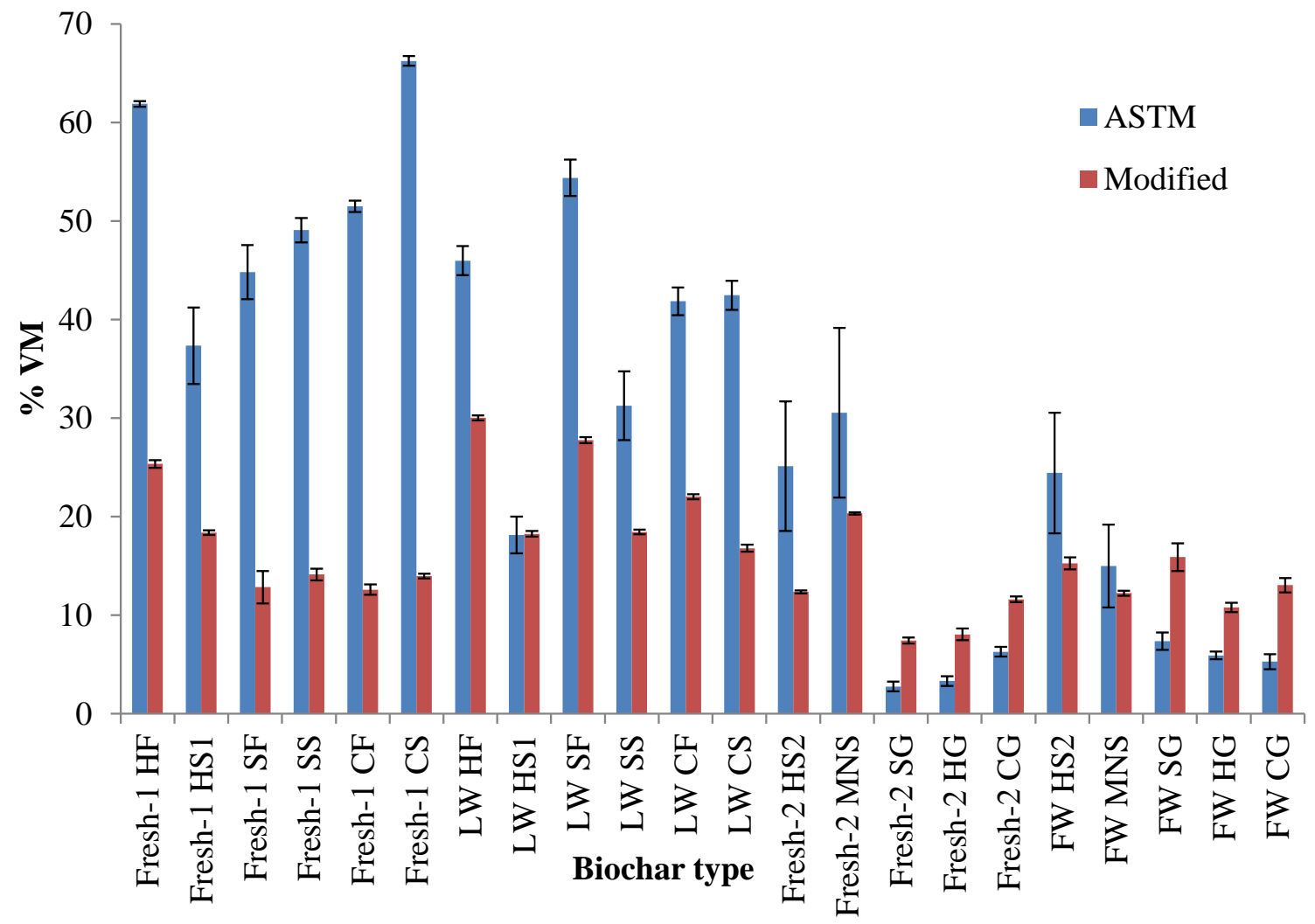


Fig. 4

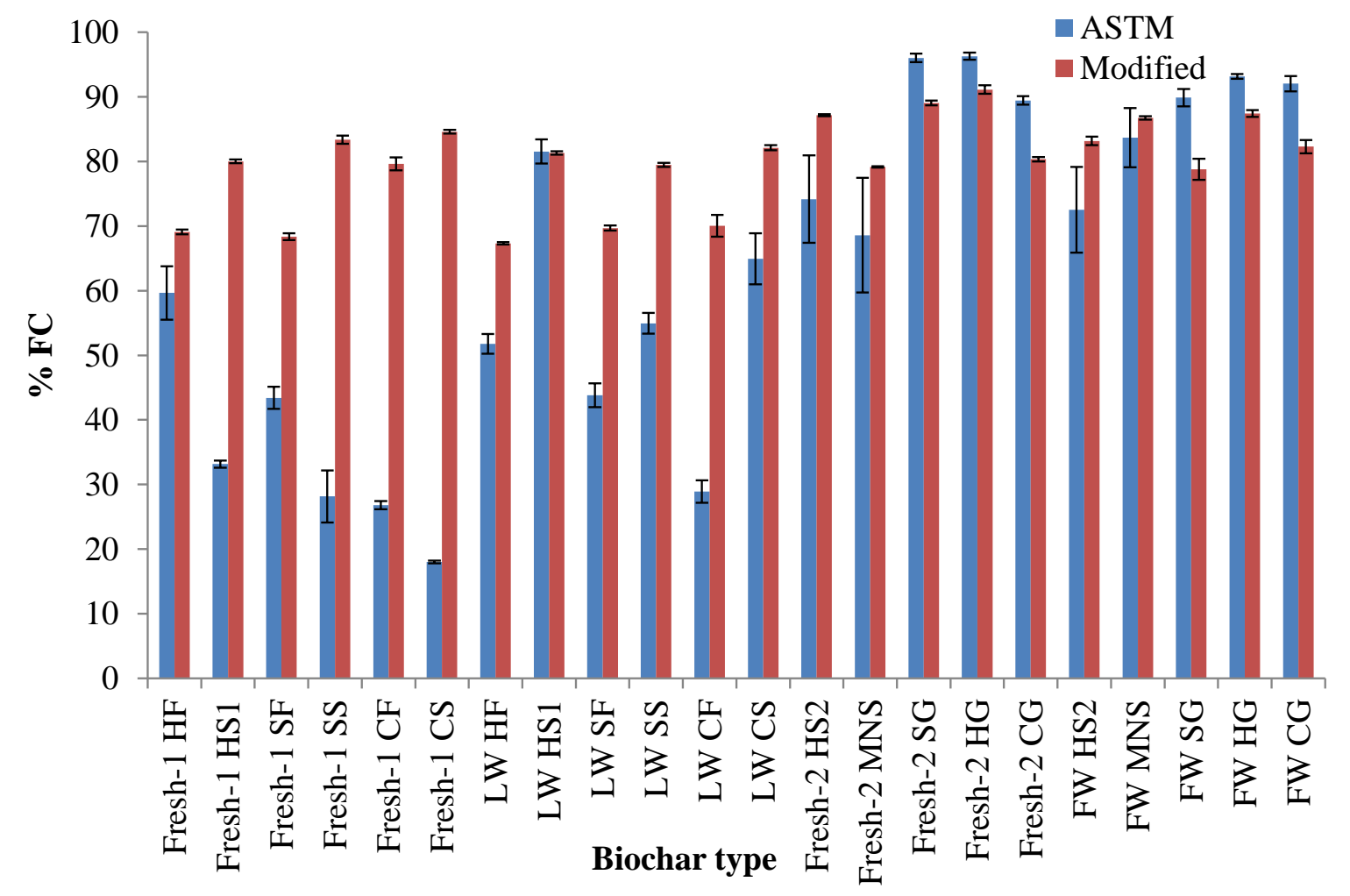


Fig. 5a

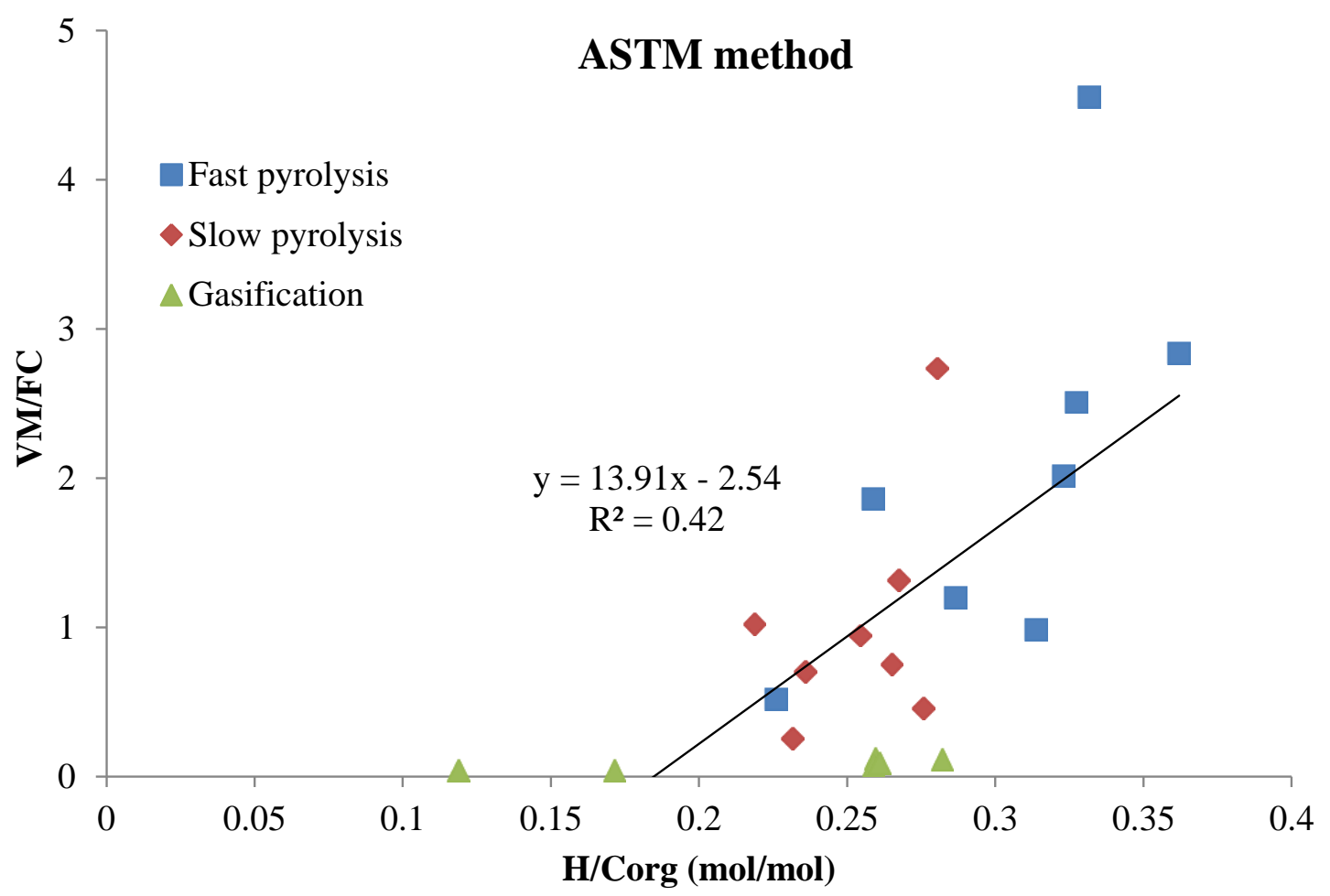


Fig. 5b

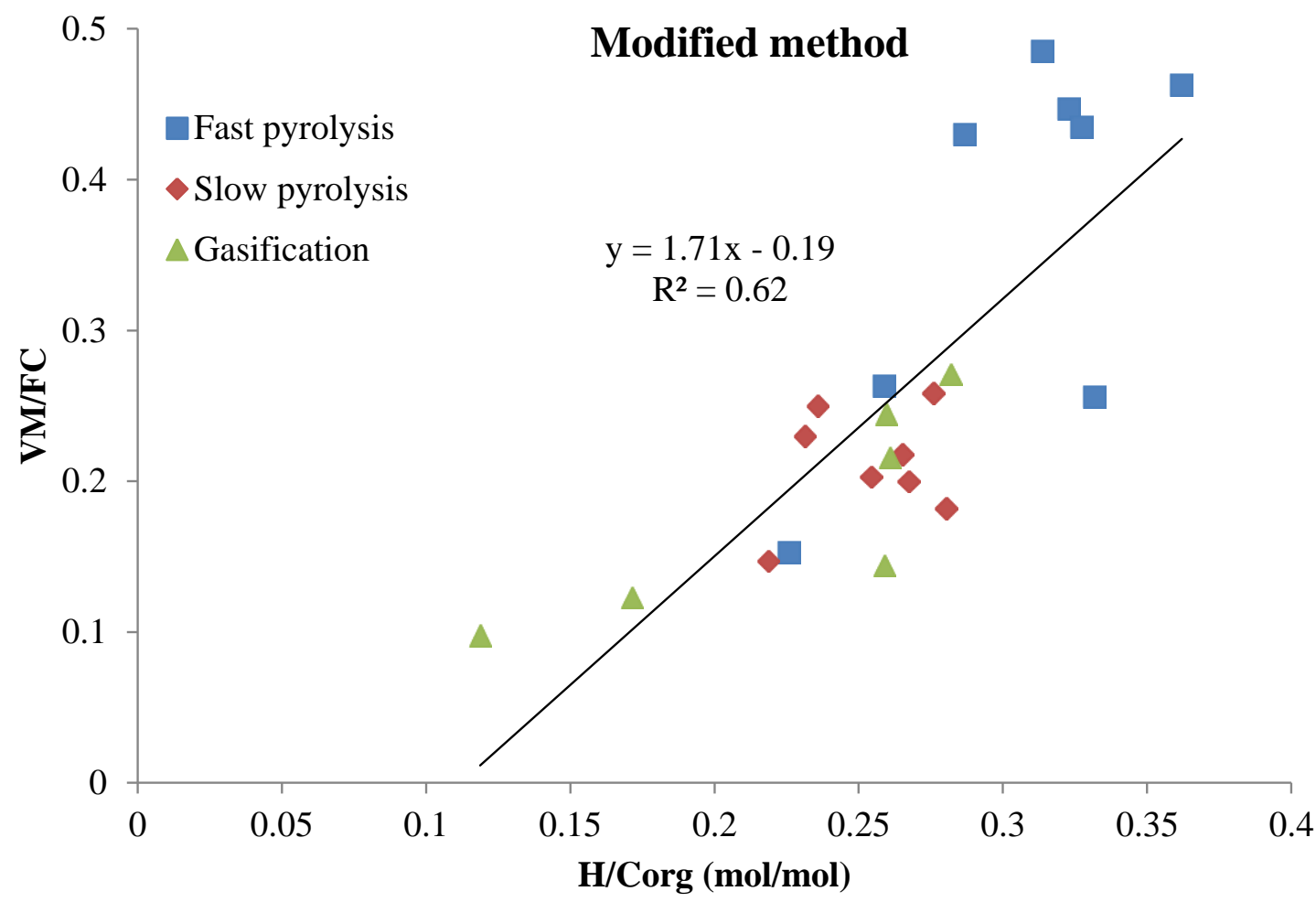

